\title{
(Convex) Level Sets Integration
}

\author{
Jean-Pierre Crouzeix · Andrew \\ Eberhard • Daniel Ralph \\ Dedicated to Vladimir Demjanov
}

Received: date / Accepted: date

\begin{abstract}
The paper addresses the problem of recovering a pseudoconvex function from the normal cones to its level sets that we call the convex level sets integration problem. An important application is the revealed preference problem. Our main result can be described as integrating a maximally cyclically pseudoconvex multivalued map that sends vectors or "bundles" of a Euclidean space to convex sets in that space. That is, we are seeking a pseudo convex (real) function such that the normal cone at each boundary point of each of its lower level sets contains the set value of the multivalued map at the same point. This raises the question of uniqueness of that function up to rescaling. Even after normalising the function long an orienting direction, we give a counterexample to its uniqueness. We are, however, able to show uniqueness under a condition motivated by the classical theory of ordinary differential equations.
\end{abstract}

Keywords Convexity and Pseudoconvexity · Monotonicity and Pseudomonotonicity $\cdot$ Maximality $\cdot$ Revealed Preferences.

Mathematics Subject Classification (2000) 26B25 $91 \mathrm{~B} 42 \cdot 91 \mathrm{~B} 16$

Jean-Pierre Crouzeix, Corresponding Author,

LIMOS, Campus Scientifique des Cézeaux, Université Blaise Pascal, 63170 Aubière, France,

E-mail: jp.crouzeix@isima.fr

Andrew Eberhard

School of Mathematical \& Geospatial Sciences, RMIT University, Melbourne, VIC., Australia, E-mail: andy.eb@rmit.edu.au

Daniel Ralph

University of Cambridge, Judge Business School, UK, E-mail: d.ralph@jbs.cam.ac.uk 


\section{Introduction}

Given a (multivalued) map the convex integration problem consists of finding a convex function whose subdifferential, or gradient in the smooth case, coincides with the map. Under appropriate conditions, the solution is unique up to a constant.

In this paper, one considers the much more difficult problem where we are seeking a pseudo convex function corresponding to a map that generates the normal cones to the level sets of the function. One question is the uniqueness of the solution up to a scalarization.

In both cases, two situations are considered: the map is continuously differentiable and the map is multivalued.

Despite the great difference of difficulties encountered, there are similarities in the treatment of the differentiable and multivalued cases in these two situations.

\section{Statement of the problem}

Given $a \in C \subset \mathbb{I}^{n}, \alpha \in \mathbb{I R}$ and $F: C \rightarrow \mathbb{I}^{n}$, the classical integration problem consists of finding $f: C \rightarrow \mathrm{IR}$ differentiable on $C$ such that $\nabla f(x)=F(x)$ for all $x \in C$ and $f(a)=\alpha$. For convenience, we assume throughout all the paper that $C$ is open and convex.

When $f$ turns out to be convex,

$$
\langle F(x), y-x\rangle \leq f(y)-f(x) \forall x, y \in C
$$

In the convex case, the generalization of the integration problem to multivalued monotone maps $F$ consists of finding $f$ convex on $C$ such that $f(a)=\alpha$ and

$$
\left\langle x^{*}, y-x\right\rangle \leq f(y)-f(x) \quad \forall x, y \in C, \forall x^{*} \in F(x) .
$$

We speak of this problem as the convex integration problem.

Another problem, significantly more difficult, consists, when $F$ is single valued, of finding $f \rightarrow$ IR differentiable such that

$$
\frac{\nabla f(x)}{\|\nabla f(x)\|}=\frac{F(x)}{\|F(x)\|} \quad \forall x \in C
$$

We speak of this problem as the differentiable level sets integration problem.

In case where $f$ turns out to be pseudoconvex, then

$$
\langle\nabla f(x), y-x\rangle \leq 0 \quad \forall x \in C, \quad \forall y \in S(x)
$$


where $S(x)$ is the level set

$$
S(x):=\{y \in C: f(y) \leq f(x)\}
$$

We consider the generalisation of this problem to the case where $F$ is multivalued. It consists of finding $f$ pseudoconvex such that

$$
\left\langle x^{*}, y-x\right\rangle \leq 0 \quad \forall x \in C, \forall y \in S(x), \forall x^{*} \in F(x)
$$

We call this problem the convex level sets integration problem.

If the theories of classical and convex integration have been well established over several decades, this is not yet the case for level sets integration. However, despite the different levels of difficulties inherent in these problems, the approaches of their respective treatments present strong similarities. It is but one of the purposes of this paper to show the similarities and the differences between differentiable and non differentiable, convex and convex level sets integration. In this respect, Section 3 briefly discusses classical integration in order to prepare the treatment of (convex) level sets integration. Section 4, based on Crouzeix-Rapcsák [1], treats the differentiable level sets convex problem for comparison with Section 6 where the nondifferentiable case is studied. Section 5 is a very short review of the generalized convex and monotone properties used in Section 6. Finally, Section 7 connects the paper with the problem known in economics as the revealed preference problem.

The inner product of two vectors $x, y \in \mathbb{I}^{n}$ is denoted by $\langle x, y\rangle$ or $x^{\top} y$. The Euclidean norm of a vector $x \in \mathrm{IR}^{n}$ is denoted by $\|x\|$. The convex hull of a set $A$ is denoted by conv $(A)$. Given a multivalued map $F: \mathbb{I}^{n} \rightrightarrows \mathbb{I}^{n}$ its graph and its domain are the sets $\operatorname{graph}(F):=\left\{\left(x, x^{*}\right) \in \mathbb{I}^{n} \times \mathbb{R}^{n}\right.$ : $\left.x^{*} \in F(x)\right\}$ and $\operatorname{dom}(F):=\left\{x \in \mathbb{I}^{n}: F(x) \neq \emptyset\right\}$ respectively. For a set $S \subset \mathbb{I}^{n} \times \mathbb{I}^{n}$, Proj $(\operatorname{graph}(F)):=\left\{x \in \mathbb{I}^{n}: \exists y \in \mathbb{R}^{n},(x, y) \in S\right\}$, e.g., $\operatorname{dom}(F)=\operatorname{Proj}(\operatorname{graph}(F))$.

\section{Integration and Convex Integration}

Through all sections $C \subset \mathrm{IR}^{n}$ is an open convex set.

\subsection{The Differentiable Case}

Assume that $F: C \rightarrow \mathrm{IR}$ is continuous. Let $a \in C$, and $\alpha \in \mathrm{IR}$. The problem consists in finding a function $f: C \rightarrow \mathrm{IR}$ continuously differentiable on $C$ such that $\nabla f(x)=F(x)$ for all $x \in C$ and $f(a)=\alpha$. 
If such a $f$ exists, then necessarily,

$$
f(x)=\alpha+\int_{0}^{1}\langle F(a+t(x-a)), x-a\rangle d t \quad \forall x \in C .
$$

Thus, if the problem admits one solution, the solution is unique. The existence of a solution is a little more difficult question, additional assumptions are required. In order to prepare the treatment of differentiable level sets integration in Section 3, we briefly describe a sketch of the proof. The function $f$ given by formula (1) is a solution of the problem if and only if for all $d$ such that $(a+d) \in C$

$$
\frac{1}{\|h\|}[f(a+d+h)-f(a)-\langle F(a+d), h\rangle] \rightarrow 0 \quad \text { when } h \rightarrow 0 .
$$

It is well known that this condition holds as soon as $F$ is continuously differentiable on $C$ and the matrix $F^{\prime}(x)$ is symmetric for all $x \in C$. Under this condition, $f$ is convex if and only if $F^{\prime}(x)$ is positive semidefinite for all $x \in C$. Expression (1) provides a construction of the (convex) function $f$.

To summarize: the existence of $f$ such that $F$ coincides with $\nabla f$ is ensured by the continuity of $F^{\prime}$ and the symmetry of the matrices $F^{\prime}(x)$, the convexity of $f$ by the positive semidefiniteness of the matrices $F^{\prime}(x)$, the uniqueness of $f$ up to a constant is ensured by the continuity of $F$.

\subsection{The Nondifferentiable Case}

Next, we consider the generalization of the problem to nondifferentiable functions $f$. An appropriate generalization of differentiability for convex functions is the subdifferentiability in the sense of Fenchel. Recall that the subdifferential $\partial f$ of a function $f$ is defined by

$$
\partial f(x):=\left\{x^{*}:\left\langle x^{*}, y-x\right\rangle \leq f(y)-f(x) \forall y\right\} .
$$

The subdifferential of a convex function is a cyclically monotone map, i.e., for all finite family $\left\{\left(x_{i}, x_{i}^{*}\right), i=0, \cdots, p\right\} \subset \operatorname{graph}(\partial f)=\left\{\left(x, x^{*}\right): x^{*} \in \partial f(x)\right\}$ with $\left(x_{0}, x_{0}^{*}\right)=\left(x_{p}, x_{p}^{*}\right)$ one has

$$
\sum_{i=0}^{p-1}\left\langle x_{i}^{*}, x_{i+1}-x_{i}\right\rangle \leq 0 .
$$

If $f: C \rightarrow \mathrm{IR}$ is a differentiable convex function, then $\partial f(x)=\{\nabla f(x)\}$ at all $x \in C$. 
The convex integration problem consists in building a convex function $f$ from a cyclically monotone multivalued map $F: C \rightrightarrows \mathbb{I}^{n}$ such that $f(a)=\alpha$ and

$$
F(x) \subset \partial f(x) \forall x \in \operatorname{dom}(F):=\left\{y: \exists y^{*} \text { such that } y^{*} \in F(x)\right\}
$$

\subsubsection{Existence}

Let $\emptyset \neq S \subset \operatorname{graph}(F),\left(a, a^{*}\right) \in S$ and $\alpha \in \mathrm{IR}$. The following construction of $f_{S}: \mathrm{IR}^{n} \rightarrow \mathrm{IR} \cup\{+\infty\}$ is borrowed from Rockafellar [2]:

$$
f_{S}(x):=\alpha+\sup \left[\begin{array}{lc}
p & p \in \mathbb{N}, x_{p+1}=x, \\
\sum_{i=0}^{p}\left\langle x_{i}^{*}, x_{i+1}-x_{i}\right\rangle: & \left(x_{0}, x_{0}^{*}\right)=\left(a, a^{*}\right), \\
\left(x_{i}, x_{i}^{*}\right) \in S, i=1, \cdots, p
\end{array}\right] .
$$

It straightforwardly follows that $f_{S}$ is a proper convex lower semicontinuous function because it is a supremum of affine functions. Also, $f_{S} \leq f_{T}$ whenever $S \subset T \subset \operatorname{graph}(F)$. Next, due to the cyclic monotonicity of $F$, one has $f_{S}(a)=\alpha$ and $x^{*} \in \partial f_{S}(x)$ for all $\left(x, x^{*}\right) \in S$. Actually, $f_{S}$ is the smallest lower semicontinuous convex function for which these two conditions hold. Also, $f_{S}$ is finite on the interior of the convex hull of $\operatorname{Proj}(S)$.

If card $(\mathrm{S})$ is finite, then $f_{S}$ is a convex piecewise linear function. It can be explicitly computed through a finite number of steps.

Assume that $\operatorname{Proj}(S)=\operatorname{dom}(F)$. Set $f=f_{S}$. We have shown the existence of a convex lower semicontinuous function $f$ on $C$ such that $f(a)=\alpha$ and $F(x) \subset \partial f(x)$ for all $x \in \operatorname{dom}(F)$.

\subsubsection{Uniqueness}

Next, assume that the closure of $\operatorname{dom}(F)$ is convex and coincides with the closure of $\operatorname{Proj}(S)$, then $f_{S}$ is the unique convex lower semicontinuous function $f$ such that $f(a)=\alpha$ and $F(x) \subset \partial f(x)$ for all $x \in \operatorname{Proj}(S)$. Moreover, for all $x$ in the interior of the convex hull of $\operatorname{dom}(F), \partial f(x)$ is the closed convex hull of the set

$$
F_{S}(x):=\left\{x^{*}: \exists \text { a sequence }\left\{\left(x_{k}, x_{k}^{*}\right)\right\}_{k \in \mathbb{N}} \subset S \text { converging to }\left(x, x^{*}\right)\right\} .
$$

Assume in addition the set $S \subset \operatorname{graph}(F)$ is a countable set:

$$
S:=\left\{\left(x_{0}, x_{0}^{*}\right),\left(x_{1}, x_{1}^{*}\right), \cdots,\left(x_{k}, x_{k}^{*}\right), \cdots\right\}_{k \in \mathbb{N}} .
$$

Let us consider the sets

$$
S_{k}:=\left\{\left(x_{0}, x_{0}^{*}\right),\left(x_{1}, x_{1}^{*}\right), \cdots,\left(x_{k}, x_{k}^{*}\right)\right\}
$$


and the associated functions $f_{k}=f_{S_{k}}$. Then $f_{k} \leq f_{k+1}$ for all $k$. The function $f$ defined by $f(x)=\lim _{k \rightarrow+\infty} f_{k}(x)$ for all $x \in C$ is the unique solution of the problem. Because the computation of the function $f_{k}$ is achieved through a finite number of steps, the sequence $\left\{f_{k}\right\}$ provides efficient numerical approximations of the function $f$.

\subsubsection{Is F a Subdifferential?}

Our last question concerns the equality $F(x)=\partial f(x)$ for all $x \in C$. It results from above that $F$ coincides with $\partial f$ on the convex open set $C$ if and only if $F$ is cyclically monotone, $\operatorname{dom}(F)=C$ and, for all $x \in C, F(x)$ coincides with the closed convex hull of the set

$$
\left\{x^{*}: \exists \text { a sequence }\left\{\left(x_{k}, x_{k}^{*}\right)\right\}_{k \in \mathbb{N}} \subset \text { graph }(F) \text { converging to }\left(x, x^{*}\right)\right\} \text {. }
$$

This continuity property is equivalent to say that $F$ is maximal monotone on $C$, see [3] for instance. The following formula (to be compared with (1))

$$
f(x)=\alpha+\int_{0}^{1}\left\langle x^{*}(t), x-a\right\rangle d t \forall x \in C,
$$

where $x^{*}(t)$ is arbitrarily chosen in $F(a+t(x-a))$, gives an expression of $f(x)$ in terms of an integral on a real line. Due to the monotonicity and the continuity properties of the subdifferential, $f$ is well and uniquely defined by formula (5).

To summarize: the existence and the convexity of $f$ is ensured by the cyclic monotonicity of $F$, the uniqueness of $f$ by a density property, the coincidence of $F$ with $\partial f$ by the maximal monotonicity of $F$.

\section{The Differentiable Level Sets Integration Problem}

This section deals with the existence of a function $f: C \rightarrow \mathrm{IR}$ twice continuously differentiable such that in a neighborhood of of a point $a \in C$

$$
\frac{\nabla f(x)}{\|\nabla f(x)\|}=\frac{F(x)}{\|F(x)\|}
$$

where $F: C \rightarrow \mathrm{IR}^{n}$ is continuous differentiable and does not vanish on the open set $C$. In contrast with the previous section, only the direction of the gradient of $f$ is given, not the gradient itself. Hence, it is no more sufficient to fix the value of the function at some point $a$ to determine properly $f$ : if $f$ is one solution of the problem then $k \circ f$, with $k$ differentiable strictly increasing, is also a solution. 
We shall construct a local solution to the problem. Actually, we shall show that, under appropriate conditions, given $a \in C$, there exists one function $f$, unique up to a scalarisation function $k$, which responds to the question over an open convex neighborhood of $a$.

Since $F(a) \neq 0$ and $F$ is continuous, there exist $e \in \mathbb{I}^{n}$ with $\|e\|=1$, a convex neighborhood $E$ of $e$ and a convex neighborhood $V$ of $a$ such that $\left\langle x^{*}, F(x)\right\rangle>0$ for all $x \in V, x^{*} \in E$. Without loss of generality, we can assume that $e^{\top} F(x)=1$ for all $x \in V$. The problem is rewritten as: Find $f$ twice differentiable on $V$ such that

$$
\frac{\nabla f(x)}{e^{\top} \nabla f(x)}=F(x) \forall x \in V .
$$

It follows that necessarily

$$
F^{\prime}(x)\left[I-e^{\top} F(x)\right]=\left[I-F(x) e^{\top}\right] \frac{\nabla^{2} f(x)}{e^{\top} \nabla f(x)}\left[I-e^{\top} F(x)\right] \forall x \in V .
$$

A necessary condition for the existence of a solution is the symmetry of the matrix $F^{\prime}(x)\left[I-e^{\top} F(x)\right]$. Then the matrix $F^{\prime}(x)$ is symmetric on the orthogonal space to $F(x)$.

\subsection{If a Solution Exists}

Suppose that $f$ is the solution of the problem, then $f(x+t d)>f(x)$ for all $x \in V, d \in \mathbb{I R}^{n}$ and $t>0$ such that $x+t d \in V$. Given $x \in V$, we consider its level sets

$$
S(x):=\left\{x^{\prime} \in V: f\left(x^{\prime}\right) \leq f(x)\right\}, C(x)=\left\{x^{\prime} \in V: f\left(x^{\prime}\right)=f(x)\right\} .
$$

These sets depend only on $x$, they are the same for all functions of the form $k \circ f$ with $k$ differentiable and continuous. Our approach consists of determining these sets, in fact in constructing the indifference surfaces $C(x)$.

There exists a $n \times(n-1)$ matrix $A$ such that $A^{\top} A$ is the $(n-1) \times(n-1)$ identity matrix and $A^{\top} e=0$. Any $x \in \mathbb{R}^{n}$ admits one unique decomposition of the form

$$
x:=a+A y-s e, \quad y \in \mathrm{IR}^{n-1}, s \in \mathrm{IR} .
$$

In a neighborhood of $a$ the indifference curves are described through functions $g_{\lambda}$ such that for $(y, \lambda)$ close enough to the origin of $\mathbf{I R}^{n}$,

$$
f(a+\lambda e)=f\left(a+A y-g_{\lambda}(y) e\right) .
$$


In view of the implicit function theorem, $g_{\lambda}$ is the unique solution of the partial difference equation problem: Find $g_{\lambda}$ differentiable such that $g_{\lambda}(0)=-\lambda$ and

$$
\nabla g_{\lambda}(y)=\frac{A^{\top} \nabla f\left(a+A y-g_{\lambda}(y) e\right)}{e^{\top} \nabla f\left(a+A y-g_{\lambda}(y) e\right)}=A^{\top} F\left(a+A y-g_{\lambda}(y) e\right) \quad \forall y \in V .
$$

Equivalently, $g_{\lambda}$ is uniquely defined by the implicit formula

$$
g_{\lambda}(y):=-\lambda+\int_{0}^{1}\left\langle A^{\top} F\left(a+t A y-g_{\lambda}(t y) e\right), y\right\rangle d t .
$$

If the problem admits solutions, the level sets $S(x)$ and the indifference curves $C(x)$ are uniquely defined. Hence the functions $f$ which are solutions are defined up to a scalarization.

\subsection{Existence of a Solution}

Through this subsection we assume that there exists a convex compact neighborhood $V$ of $a$ such that

A1: $e^{\top} F(x)=1$ for all $x \in V$,

A2: $F$ is continuously differentiable on $V$,

A3: $F^{\prime}(x)\left[I-e F^{\top}(x)\right]$ is a symmetric matrix for all $x \in V$.

It is easy to see that $\mathbf{A} \mathbf{3}$ is equivalent to:

A4: $h^{\top} F^{\prime}(x) k=k^{\top} F^{\prime}(x) h \quad \forall x \in V, \forall h, k \perp F(x)$.

Let $g$ be the function given by the implicit equation

$$
g(y, \lambda)=-\lambda+\int_{0}^{1}\langle F(a+t A y-g(t y, \lambda) e), A y\rangle d t .
$$

We shall show that $g$ is differentiable in a neighborhood of $(0,0)$. Actually, we shall find $\nabla_{y} g(y, \lambda) \in \mathbb{I}^{n-1}$ and $g_{\lambda}^{\prime}(y, \lambda) \in \mathbb{I R}$ such that

$$
\frac{g(y+z, \lambda+\mu)-g(y, \lambda)-\left\langle\nabla_{y} g(y, \lambda), z\right\rangle-\mu g_{\lambda}^{\prime}(y, \lambda)}{\|(z, \mu)\|} \rightarrow 0 \text { when }\|(z, \mu)\| \rightarrow 0 .
$$

We just give a sketch of the proof in focusing on the places where the assumptions are used. For a detailed proof see [1]. The quantities $y, z, \lambda, \mu$ being fixed, let us introduce

$$
\begin{aligned}
x_{1}(t) & :=a+t A y+g(t y, \lambda) e, \\
x_{2}(t) & :=a+t A y+t A z-g(t y+t z, \lambda+\mu) e, \\
\delta(t) & :=g(t y+t z, \lambda+\mu)-g(t y, \lambda)-t F^{\top}\left(x_{1}(t)\right) A z .
\end{aligned}
$$


Then,

$$
\begin{aligned}
x_{1}^{\prime}(t) & =\left[I-e F^{\top}\left(x_{1}(t)\right)\right] A y, \\
x_{2}(t)-x_{1}(t) & =t\left[I-e F^{\top}\left(x_{1}(t)\right)\right] A z-\delta(t) e .
\end{aligned}
$$

Given $x, h \in \mathbb{I R}^{n}$ such that $x, x+h \in V$, let us consider the function defined by

$$
E(x, x+h)= \begin{cases}0 & \text { if } h=0, \\ \frac{1}{\|h\|}\left[F(x+h)-F(x)-F^{\prime}(x) h\right] & \text { if } h \neq 0 .\end{cases}
$$

Since $F$ is continuously differentiable and $V$ is compact, $E$ is continuous and $K_{0}>0$ exists such that $\|E(x, h)\| \leq K$ for all $x, h$ such that $x, x+h \in V$. Then,

$\delta^{\prime}(t)=(A y+A z)^{\top}\left[F\left(x_{2}(t)\right)-F\left(x_{1}(t)\right)\right]-t(A z)^{\top} F^{\prime}\left(x_{1}(t)\right)\left[I-e^{\top} F\left(x_{1}(t)\right)\right] A y$.

From assumption A3,

$$
(A z)^{\top} F^{\prime}\left(x_{1}(t)\right)\left[I-e^{\top} F\left(x_{1}(t)\right)\right] A y=(A y)^{\top} F^{\prime}\left(x_{1}(t)\right)\left[I-e^{\top} F\left(x_{1}(t)\right)\right] A z . \quad \text { (9) }
$$

On the other hand,

$$
\begin{aligned}
F\left(x_{2}(t)\right)-F\left(x_{1}(t)\right)= & t F^{\prime}\left(x_{1}(t)\right)\left[I-e^{\top} F\left(x_{1}(t)\right)\right] A z-\delta(t) F^{\prime}\left(x_{1}(t)\right) e \\
& +\left\|x_{2}(t)-x_{1}(t)\right\| E\left(x_{1}(t), x_{2}(t)\right) .
\end{aligned}
$$

Hence,

$$
\begin{aligned}
\delta^{\prime}(t)= & t(A z)^{\top} F^{\prime}\left(x_{1}(t)\right)\left[I-e^{\top} F\left(x_{1}(t)\right)\right] A z-\delta(t)(A y+A z)^{\top} F^{\prime}\left(x_{1}(t)\right) e \\
& +\left\|x_{2}(t)-x_{1}(t)\right\| E\left(x_{1}(t), x_{2}(t)\right) .
\end{aligned}
$$

It can be shown (see [1]) that $K_{1}$ and $K_{2}$ exist such that $\delta(t) \leq K_{1}\|(z, \mu)\|$ and $\left\|x_{2}(t)-x_{1}(t)\right\| \leq K_{2}\|(z, \mu)\|$. Then, $K_{3}>0$ exists such that

$$
\delta^{\prime}(t)=-\delta(t)\left\langle A y, F^{\prime}\left(x_{1}(t)\right) e\right\rangle+K_{3}\|(z, \mu)\| \varepsilon(\|(z, \mu)\|)
$$

where $\varepsilon(t) \rightarrow 0$ when $t \rightarrow 0$. Since $\delta(0)=-\mu$, there exists $K>0$ such that

$$
\delta(t)=-\mu \exp \left(-\int_{0}^{s}\left\langle A y, F^{\prime}\left(x_{1}(s) e\right)\right\rangle d s\right)+K \mid(z, \mu) \| \varepsilon(\|(z, \mu)\|) .
$$

From what, we obtain the gradient of $g$ in a neighborhood of $(0,0)$ :

$$
\nabla g(y, \lambda)=\left(\begin{array}{c}
A^{\top} F(a+A y-g(y, \lambda) e) \\
-\mu \exp \left(-\int_{0}^{s}\left\langle A y, F^{\prime}\left(x_{1}(s)\right) e\right\rangle d s\right)
\end{array}\right) .
$$

Our last step consists in constructing, among the functions $f$ for which (6) holds, the one such that $f(x=a+A y-g(y, \lambda) e)=\lambda$ in a neighborhood of $a$ 
(then $f(a+\lambda e)=\lambda$ ). Other functions will be deduced via scalarisations. Let $H: \mathbb{I}^{n+1} \rightarrow \mathbb{I R}^{n}$ be defined by

$$
H(y, \lambda, \mu):=g(y, \lambda)-\mu .
$$

Since $H_{\lambda}^{\prime}(y, \lambda, \mu)=g_{\lambda}^{\prime}(y, \lambda)>0$, by the implicit function theorem, there exists a continuously differentiable function $\theta$ such that $g(y, \theta(y, \mu))-\mu=0$ for $(y, \mu)$ in a neighborhood of $(0,0)$. Next, set $f(x=a+A y-\mu e)=\theta(y, \mu)$. $f$ is continuously differentiable in $(y, \mu)$. One has

$$
\begin{gathered}
A^{\top} \nabla f(x)=\nabla_{y} \theta(y, \mu)=-\frac{\nabla_{y} g(y, \lambda)}{g_{\lambda}^{\prime}(y, \lambda)}=-\frac{A^{\top} F(x)}{g_{\lambda}^{\prime}(y, \lambda)}, \\
e^{t} \nabla f(x)=-\theta_{\mu}^{\prime}(y, \mu)=-\frac{e^{\top} F(x)}{g_{\lambda}^{\prime}(y, \lambda)} .
\end{gathered}
$$

Therefore, regarding $\nabla f(x)$ as the $n$-dimensional vector $\left(A^{\top} \nabla f(x), e^{\top} \nabla f(x)\right)$, for $x$ in a small enough neighborhood of $a$ we have

$$
\frac{\nabla f(x)}{\|\nabla f(x)\|}=\frac{F(x)}{\|F(x)\|} .
$$

4.3 Convexity of the Level Sets

The level sets $S(x)$ are convex if and only if the functions $g_{\lambda}=g(., \lambda)$ are convex. Here,

$$
\nabla g_{\lambda}(y)=A^{\top} F\left(a+A y-g_{\lambda}(y) e\right) .
$$

Because $F$ is twice continuously differentiable, the functions $g_{\lambda}$ are also twice continuously differentiable and

$$
\nabla^{2} g_{\lambda}(y)=A^{\top} F^{\prime}\left(a+A y-g_{\lambda}(y) e\right)\left(I-e^{\top} F\left(a+A y-g_{\lambda}(y) e\right)\right) A .
$$

Hence, in a convex neighborhood of $a$, the level sets are convex if and only if for $x=a+A y-\mu e$ in the neighborhood one has

$$
(A k)^{\top} F^{\prime}(x)\left(I-e^{\top} F(x)\right) A k \geq 0 \quad \forall k \in \mathbb{R}^{n-1} .
$$

Let any $h=A k-\xi e \in \mathbb{I}^{n}$. Because of $\mathbf{A 3}$, (11) is equivalent to the first order characterization of pseudomonotonicity of $F$ on $V$ [4].

A5: $h^{\top} F^{\prime}(x) h \geq 0 \quad \forall x \in V, \forall h$ such that $h^{\top} F(x)=0$.

which means that the matrix $F^{\prime}(x)$ is positive semi-definite on the orthogonal subspace to $F(x)$. 


\subsection{Remarks}

We have just given above one overview of the construction. Besides the existence of the constants $K_{1}$ and $K_{2}$, there are some very technical points which have been left. Their treatment is not too difficult but it makes the proof heavy. In particular, the construction of the quantity $g_{\lambda}(y)$ needs that, for all $t \in[0,1]$, the point $a+t A y-g_{\lambda}(t y) e$ belongs to the neighborhood. We report to Crouzeix-Rapscak [1] for a detailed proof and a global result.

To summarize: the existence of a solution $f$ of (6) is ensured by the continuity of $F^{\prime}$ and the symmetry of the matrices $F^{\prime}(x)$ on the orthogonal subspace to $F(x)$, the pseudo convexity of $f$ by the positive semidefiniteness of the matrices $F^{\prime}(x)$ on the orthogonal subspace, the uniqueness of $f$ up to a scalarization is ensured by the Lipschitz continuity of F.

\section{A Brief Survey on Quasi/Pseudo Convexity and Quasi/Pseudo Monotonicity}

Let $C$ be an open convex set and $f: C \rightarrow \mathrm{IR}$. $f$ is said to be quasiconvex on $C$ if its strict level sets $\widetilde{S}(x):=\{y \in C: f(y)<f(x)\}$ are convex. Then the level sets $S(x):=\{y \in C: f(y) \leq f(x)\}$ are also convex. If $f$ is differentiable on $C$, each one of the two following conditions is necessary and sufficient for $f$ to be quasiconvex on $C$.

$$
\begin{aligned}
& x, y \in C \text { with } f(y) \leq f(x) \Longrightarrow\langle\nabla f(x), y-x\rangle \leq 0, \\
& x, y \in C \text { with } f(y)<f(x) \Longrightarrow\langle\nabla f(x), y-x\rangle \leq 0 .
\end{aligned}
$$

For a quasiconvex function $\nabla f(x)=0$ does not implies that $f$ has a local minimum at $x$ as shown by the following function $f(x)=-(x+1)^{2}$ if $x \leq-1$, $f(x)=-0$ if $-1 \leq x \leq 1, f(x)=(x-1)^{2}$ if $x \geq 1$. A more convenient concept for optimization purposes is pseudoconvexity: $f$ differentiable on the open convex set $C$ is said to be pseudoconvex on $C$ if

$$
x, y \in C \text { with } f(y)<f(x) \Longrightarrow\langle\nabla f(x), y-x\rangle<0 .
$$

A differentiable pseudoconvex function is quasiconvex. Then, $f$ is pseudoconvex on $C$ if and only if for all $x \in C$

$$
\widetilde{S}(x)=\{y \in C: f(y)<f(x)\} \subset\{y \in C:\langle\nabla f(x), y-x\rangle<0\},
$$

or equivalently, if and only if for all $x \in C$

$$
S(x)=\{y \in C: f(y) \leq f(x)\} \subset\{y \in C:\langle\nabla f(x), y-x\rangle \leq 0\} .
$$


A second order characterization of pseudoconvex functions is as follows [5]: let $f$ be twice continuously differentiable on the open convex set $C$ with $\nabla f(x) \neq 0$ for all $x \in C$. Then $f$ is pseudoconvex on $C$ if and only if for all $x \in C$

$$
h^{\top} \nabla^{2} f(x) h \geq 0 \quad \forall h \in \mathbb{R}^{n} \text { such that } h^{\top} \nabla f(x)=0 .
$$

Let $f$ be pseudoconvex. Let any points $x_{0}, x_{1}, \cdots, x_{p} \in C$ be such that $\left\langle\nabla f\left(x_{i}\right), x_{i+1}-x_{i}\right\rangle \geq 0$ for $i=0,1, \cdots, p-1$. Then, $f\left(x_{i+1}\right) \geq f\left(x_{i}\right)$. Therefore $f\left(x_{p}\right) \geq f\left(x_{0}\right)$ and $\left\langle\nabla f\left(x_{p}\right), x_{0}-x_{p}\right\rangle \leq 0$. Moreover, if $\left\langle\nabla f\left(x_{i}\right), x_{i+1}-x_{i}\right\rangle>0$ for some $i=0,1, \cdots p-1$, then $f\left(x_{p}\right)>f\left(x_{0}\right)$ and $\left\langle\nabla f\left(x_{p}\right), x_{0}-x_{p}\right\rangle<0$. One deduces that for all finite sequence $\left\{x_{0}, x_{1}, \cdots, x_{p}\right\} \subset C$ one has

$$
\min _{i}\left[\left\langle\nabla f\left(x_{i}\right), x_{i+1}-x_{i}\right\rangle: i=0,1, \cdots, p\right] \leq 0 \text { where } x_{p+1}=x_{0},
$$

and, in case where the minimum equals 0 , all quantities $\left\langle\nabla f\left(x_{i}\right), x_{i+1}-x_{i}\right\rangle$ are null. One says that the gradient of a pseudoconvex function is cyclically pseudomonotone.

Next, let us extend pseudoconvexity to nondifferentiable functions. One of the main advantages of differentiable pseudoconvex functions over quasiconvex functions is that every local minimum is global. Geometrically, this property means that the interiors and the closures of the convex sets $\widetilde{S}(x)$ and $S(x)$ coincide as soon as soon as $f(x)>\inf f$. We adopt this property for introducing a geometric definition of pseudoconvexity. A function $f$ is said to be g-pseudoconvex [6] if it is quasiconvex and if the interiors and the closures of the convex sets $\widetilde{S}(x)$ and $S(x)$ coincide as soon as soon as the interior of $\widetilde{S}(x)$ is nonempty.

Cyclic pseudomonotonicity is generalized to multivalued maps as follows. Let $C \subset \mathbb{I}^{n}$ and $F: C \rightrightarrows \mathbb{I}^{n} . F$ is said to be cyclically quasimonotone on $C$ if for all finite sequence $\left\{\left(x_{0}, x_{0}^{*}\right),\left(x_{1}, x_{1}^{*}\right), \cdots,\left(x_{p+1}, x_{p+1}^{*}\right)\right\} \subset \operatorname{graph}(F)$ such that $\left(x_{0}, x_{0}^{*}\right)=\left(x_{p+1}, x_{p+1}^{*}\right)$ one has

$$
\min _{i}\left[\left\langle x_{i}^{*}, x_{i+1}-x_{i}\right\rangle: i=0,1, \cdots, p\right] \leq 0,
$$

and cyclically pseudomonotone on $C$ if, in addition, in case where the minimum equals 0 , all quantities $\left\langle x_{i}^{*} x_{i+1}-x_{i}\right\rangle$ are null.

Let $x \in C$, let us define the normal cones to $S(x)$ and $\widetilde{S}(x)$ as

$$
\begin{gathered}
N(x):=\left\{x^{*}:\left\langle x^{*}, y-x\right\rangle \leq 0 \quad \forall y \in S(x)\right\}, \quad N^{*}(x):=\left\{x^{*} \in N(x): x^{*} \neq 0\right\}, \\
\widetilde{N}(x):=\left\{x^{*}:\left\langle x^{*}, y-x\right\rangle \leq 0 \quad \forall y \in \widetilde{S}(x)\right\} .
\end{gathered}
$$

If $f$ is g-pseudoconvex and $\widetilde{S}(x)$ is nonempty, then $N x)$ and $\widetilde{N}(x)$ coincide. If $f$ is a differentiable pseudoconvex function and $\nabla f(x) \neq 0$, then $N(x)$ is generated by $\nabla f(x)$. The problem treated in section 4 is generalized as follows: 
Given a cyclically pseudomonotone multivalued map $\Gamma: C \rightrightarrows \mathrm{IR}^{n}$, the convex level sets integration problem consists of finding a g-pseudoconvex function $f$ such that

$$
\Gamma(x) \subset N(x) \quad \forall x \in C,
$$

where $N$ denotes the normal cone map associated to $f$. The role played by the subdifferential $\partial f$ in (3) is played here by the normal cones: if $f$ is convex, its subdifferential is cycliquement monotone, similarly, if $f$ is g-pseudoconvex on the open convex set $C$ and $\operatorname{int}(\widetilde{\mathrm{S}}(\mathrm{x})) \neq \emptyset$ for all $x \in C$, the point-to-set map $N^{*}$ associated to $f$ is cyclically pseudomonotone on $C$. The argument of the proof is an easy adaptation of the one given for $\nabla f$ just above. Indeed, given $\left(x_{i}, x_{i}^{*}\right) \in \operatorname{graph}\left(N^{*}\right), f\left(x_{i+1}\right) \leq f\left(x_{i}\right)$ implies $\left\langle x_{i}^{*}, x_{i+}-x_{i}\right\rangle \leq 0$ and $f\left(x_{i+1}\right)<f\left(x_{i}\right)$ implies $\left\langle x_{i}^{*}, x_{i+}-x_{i}\right\rangle<0$. Hence, $\left\langle x_{i}^{*}, x_{i+}-x_{i}\right\rangle \geq 0$ implies $f\left(x_{i+1}\right) \geq f\left(x_{i}\right)$ and $\left\langle x_{i}^{*}, x_{i+}-x_{i}\right\rangle>0$ implies $f\left(x_{i+1}\right)>f\left(x_{i}\right)$. Proceed as for $\nabla f$.

Besides its cyclic monotonicity property the subdifferential enjoys some fundamental continuity properties. Similar properties hold for the normal cones but, because cones are unbounded by definition, we shall express these properties in terms of generators of the cones. The following results are proved in $[6]$.

Assume that $f$ is a lower semicontinuous g-pseudoconvex function on the open convex set $C$ and $a \in C$ is such that $f(a)>\inf [f(x): x \in C]$. Then, there exist $\omega \in \mathbb{I}^{n}$, a closed convex cone $K$ with nonempty interior, a convex open neighborhood $V \subset C \cap[\omega+\operatorname{int}(\mathrm{K})]$ of $a$ such that $f(y)<f(x)$ for all $x, y \in V$ with $x-y \in \operatorname{int}(\mathrm{K})$. It follows that $N(x) \subset K^{+}$where $K^{+}$is the positive dual cone of $K$. Moreover, for all $e \in \operatorname{int}(\mathrm{K})$, all $x \in V$ and all $0 \neq x^{*} \in N(x)$ one has $\left\langle x^{*}, e\right\rangle>0$. Set

$$
\Gamma(x):=\left\{x^{*} \in N(x):\left\langle x^{*}, e\right\rangle=1\right\}, \quad x \in V .
$$

Then $\Gamma(x)$ generates $N(x)$ in the sense that

$$
N(x)=\left\{\lambda x^{*}: \lambda \geq 0, x^{*} \in \Gamma(x)\right\} .
$$

$\Gamma$ is cyclically pseudomonotone. $\Gamma(x)$ is closed convex and bounded for all $x \in V$.

Let us go to the continuity properties of $\Gamma$. Let some $S \subset V$. Say that $x^{*} \in \Gamma_{S}(x)$ if there exists a sequence $\left\{x_{k}, x_{k}^{*}\right\}$ converging to $\left(x, x^{*}\right)$ with $x_{k} \in S$ and $x_{k}^{*} \in \Gamma\left(x_{k}\right)$ for all $k$. Next, let $\Gamma_{S}^{c}(x)$ be the closed convex hull of $\Gamma_{S}(x)$. Then $\Gamma(x)$ coincides with $\Gamma_{S}^{c}(x)$ on $V$ as soon as $\operatorname{cl}(S)=\operatorname{cl}(V)$. This property is equivalent to say that $\Gamma$ is maximal pseudomonotone on $V$ in the sense there is no other pseudomonotone map $\Sigma$ containing $\Gamma$ such that $\left\langle e, x^{*}\right\rangle=1$ for all 
$x \in V$ and $x^{*} \in \Sigma(x)$. As seen in Section 2, a similar property holds for maximal monotone maps.

\section{Convex Level Sets Integration}

In this section we are concerned with the local existence and uniqueness up to a scalarisation of a solution to problem (14). In line with the last section, we assume throughout all the section that $\omega \in \mathrm{IR}^{n}, K \subset \mathrm{IR}^{n}$ is an open convex cone, $V \subset \omega+K$ is an open convex neighborhood of $a \in \mathrm{IR}^{n}$ and $\Gamma$ is a maximal cyclically pseudomonotone map on $V$ such that

$$
\emptyset \neq \Gamma(x) \subset K^{+} \cap\left\{x^{*}:\left\langle x^{*}, e\right\rangle=1\right\} \quad \forall x \in V
$$

where $e=\|a-\omega\|^{-1}(a-\omega)$. Without loss of generality, we assume that $a=0$ and $V$ is of the form

$$
V=\left[\lambda_{-} e+K\right] \cap\left[\lambda_{+} e-K\right] \text { where } \lambda_{-}<0<\lambda_{+} \text {. }
$$

As in subsection 4.1, $A$ is an matrix such that $A^{\top} A$ is the $(n-1) \times(n-1)$ identity matrix and $A^{\top} e=0$. Any $x \in \mathrm{IR}^{n}$ admits one unique decomposition of the form

$$
x=A y+s e, \quad y \in \mathbb{I R}^{n-1}, s \in \mathbb{I R} .
$$

The purpose of this section is to build a quasiconvex (g-pseudoconvex) function $f$ on $V$ such that for all $x \in V$

$-(\mathrm{PM} 1) f(\lambda e)=\lambda$ for all $\lambda \in\left[\lambda_{-}, \lambda_{+}\right]$,

$-(\mathrm{PM} 2) \Gamma(x) \subset \tilde{N}(x, f)=\left\{x^{*}:\left\langle x^{*}, y-x\right\rangle \leq 0\right.$ whenever $\left.f(y)<f(x)\right\}$,

\subsection{Sandwich Inequalities}

In this subsection we improve some results of [7]. With $x \in V$, let us associate the set

$$
J(x):=\left\{\begin{array}{cc}
\exists\left(x_{0}, x_{0}^{*}\right),\left(x_{1}, x_{1}^{*}\right), \cdots,\left(x_{p}, x_{p}^{*}\right) \in \operatorname{graph}(\Gamma), \\
y \in V: \quad\left\langle x_{i}^{*}, x_{i+1}-x_{i}\right\rangle \geq 0, i=0,1, \cdots, p-1 \\
x_{0}=x, x_{p}=y, x_{i} \in V, i=0,1, \cdots, p .
\end{array}\right\}
$$

Lemma 6.1 For any $\left(x, x^{*}\right),\left(y, y^{*}\right) \in \operatorname{graph}(\Gamma)$ it holds,

1. $y \in J(x) \Longrightarrow J(y) \subset J(x)$,

2. $y \in J(x) \Longrightarrow\left\langle y^{*}, x-y\right\rangle \leq 0$,

3. $y \in J(x)$ together with $\left\langle y^{*}, x-y\right\rangle=0 \Longrightarrow\left\langle x^{*}, x-y\right\rangle=0$. 
Proof: 1) is a direct consequence of the definition of $J(x)$. Next, 2) follows from the cyclic quasimonotonicity of $\Gamma, 3)$ is a consequence of the the cyclic pseudomonotonicity.

Next, define the functions

$$
\begin{aligned}
& f_{-}(x):=\sup \left[t \in\left[\lambda_{-}, \lambda_{+}\right]: x \in J(t e)\right], \\
& f_{+}(x):=\inf \left[t \in\left[\lambda_{-}, \lambda_{+}\right]: t e \in J(x)\right] .
\end{aligned}
$$

Theorem 6.1 For any $\left(x, x^{*}\right),\left(y, y^{*}\right) \in \operatorname{graph}(\Gamma)$ it holds,

1. $\left\langle x^{*}, y-x\right\rangle \geq 0 \Longrightarrow f_{+}(x) \leq f_{+}(y)$,

2. $\Gamma(x) \subset \widetilde{N}\left(x, f_{+}\right)$,

3. $f_{+}$is quasiconvex on $V$,

4. $\lambda_{-} \leq f_{+}(x) \leq f_{+}(y) \leq \lambda_{+}$for all $x, y \in V$ with $y-x \in V$,

5. $f_{+}(\lambda e)=\lambda$ for all $\lambda \in\left[\lambda_{-}, \lambda_{+}\right]$,

6. $\left\langle x^{*}, y-x\right\rangle=\left\langle y^{*}, x-y\right\rangle=0$ implies $f_{+}(x)=f_{+}(y)$.

Proof: 1) and 2): $\left\langle x^{*}, y-x\right\rangle \geq 0 \Longrightarrow y \in J(x) \Longrightarrow J(y) \subset J(x)$. Hence, $f_{+}(x) \leq f_{+}(y)$.

3) Let $x, y \in V$ and $t \in(0,1)$. Let $z=x+t(y-x)$ and $z^{*} \in \Gamma(z)$. Either $\left\langle z^{*}, x-z\right\rangle \geq 0$ and therefore $f_{+}(z) \leq f_{+}(x)$ or $\left\langle z^{*}, y-z\right\rangle \geq 0$ and therefore $f_{+}(z) \leq f_{+}(y)$.

4) $y-x \in K$ implies $\left\langle x^{*}, y-x\right\rangle \geq 0$ which implies $f_{+}(x) \leq f_{+}(y)$. By construction of $V\left\langle x^{*}, \lambda e-x\right\rangle=0$ implies $\lambda_{-} \leq \lambda \leq \lambda_{+}$.

5) $\lambda e \in J(\lambda e)$ and therefore $f_{+}(\lambda e) \leq \lambda$. Assume for contradiction that $f_{+}(\lambda e)<\lambda$. There are $\mu$ such that $\left(x_{0}, x_{0}^{*}\right),\left(x_{1}, x_{1}^{*}\right), \cdots,\left(x_{p}, x_{p}^{*}\right)$ in graph $(\Gamma)$ such that $x_{0}=\lambda e, x_{p}=\mu e,\left\langle x_{i}^{*}, x_{i+1}-x_{i}\right\rangle \geq 0$ for $i=0, \cdots, p-1$. Let $z^{*} \in \Gamma(\mu e)$. Then, $\left\langle z^{*}, \lambda e-\mu e\right\rangle \leq 0$ which is not possible because $\left\langle z^{*}, e\right\rangle>0$.

$6)$ is a consequence of 1$)$.

Theorem 6.2 For any $\left(x, x^{*}\right),\left(y, y^{*}\right) \in \operatorname{graph}(\Gamma)$ it holds,

1. $\left\langle x^{*}, y-x\right\rangle \geq 0 \Longrightarrow f_{-}(x) \leq f_{-}(y)$,

2. $\Gamma(x) \subset \tilde{N}\left(x, f_{-}\right)$,

3. $f_{-}$is quasiconvex on $V$,

4. $\lambda_{-} \leq f_{-}(x) \leq f_{-}(y) \leq \lambda_{+}$for all $x, y \in V$ with $y-x \in V$,

5. $f_{-}(\lambda e)=\lambda$ for all $\lambda \in\left[\lambda_{-}, \lambda_{+}\right]$,

6. $\left\langle x^{*}, y-x\right\rangle=\left\langle y^{*}, x-y\right\rangle=0$ implies $f_{-}(x)=f_{-}(y)$.

Proof: 1) and 2) Let any $\lambda<f_{-}(x)$, there exists $t>\lambda$ such that $x \in J(t e)$. This added to $\left\langle x^{*}, y-x\right\rangle \geq 0$ implies $y \in J(t e)$ and therefore $\lambda<f_{-}(y)$. The two results follow.

The proofs of 3$), 4$ ) and 6) are similar to those in Theorem 6.1. 
5) $\lambda e \in J(\lambda e)$ and therefore $f_{-}(\lambda e) \geq \lambda$. Assume for contradiction that $f_{-}(\lambda e)>\lambda$. There exist $\mu>\lambda,\left(x_{0}, x_{0}^{*}\right),\left(x_{1}, x_{1}^{*}\right), \cdots,\left(x_{p}, x_{p}^{*}\right)$ in graph $(\Gamma)$ such that $x_{0}=\lambda e, x_{p}=\mu e,\left\langle x_{i}^{*}, x_{i+1}-x_{i}\right\rangle \geq 0$ for $i=0, \cdots, p-1$. Let $z^{*} \in \Gamma(\mu e)$. Then, $\left\langle z^{*}, \lambda e-\mu e\right\rangle \leq 0$ which is not possible because $\left\langle z^{*}, e\right\rangle>0$.

Theorem 6.3 (Sandwich Inequalities) Let $f$ be a quasiconvex function such that (PM1) and (PM2) hold. Then,

$$
f_{-}(x) \leq f(x) \leq f_{+}(x) \quad \forall x \in V \text {. }
$$

Proof: Let $\left(x_{i}, x_{i}^{*}\right) \in \operatorname{graph}(\Gamma), i=0, \cdots, p$ such that $\left\langle x_{i}^{*}, x_{i+1}-x_{i}\right\rangle \geq 0$ for $i=0, \cdots p-1$. Then $f\left(x_{0}\right) \leq f\left(x_{1}\right) \leq \cdots \leq f\left(x_{p}\right)$. Set $x_{0}=\lambda e$ and $x_{p}=x$. Then, $x \in J(\lambda e)$ and $f(x) \geq \lambda$. Therefore, $f(x) \geq f_{-}(x)$. Set $x_{p}=\lambda e$ and $x_{0}=x$. Then, $\lambda e \in J(x), f(x) \leq \lambda$ and $f(x) \leq f_{+}(x)$.

The cyclic pseudomonotonicity and the maximal pseudomonotonicity of $\Gamma$ do not guarantee the existence of a g-pseudoconvex function $f$ associated with $\Gamma$ as shown in the following counter-example:

Example 6.1 Let $g: \mathrm{IR}^{2} \rightarrow \mathrm{IR}$ be defined by

$$
g(x):= \begin{cases}x_{2}, & \text { if } x_{2} \geq 0, x \neq(0,0), \\ -1, & \text { if } x=(0,0), \\ x_{2} x_{1}^{-2}, & \text { if }-x_{1}^{2} \leq x_{2}<0, \\ x_{2}+x_{1}^{2}-1, & \text { if } x_{2}+x_{1}^{2} \leq 0 .\end{cases}
$$

$g$ is g-pseudoconvex and lower semicontinuous on $\mathrm{IR}^{2}$, it is not continuous at $(0,0)$. Take $e=(0,1)^{\top}$ and define $\Gamma: \mathrm{IR}^{2} \rightarrow \mathrm{IR}^{2}$ by

$$
\Gamma(x):= \begin{cases}(0,1)^{\top} & \text { if } x_{2} \geq 0, \\ \left(-2 x_{2} x_{1}^{-1}, 1\right)^{\top} & \text { if }-x_{1}^{2} \leq x_{2}<0, \\ \left(2 x_{1}, 1\right)^{\top} & \text { if } x_{2}+x_{1}^{2} \leq 0 .\end{cases}
$$

It is easy to see that $\Gamma(x)$ generates the normal cone $N(x)$ and $\tilde{N}(x)$ at $x$ to the level sets $S(x)$ and $\widetilde{S}(x)$ of $g$. The map $\Gamma$ is cyclically pseudomonotone and continuous on $\mathrm{IR}^{2}$ and thereby maximal cyclically pseudomonotone on 
this set. The functions $f_{-}, f_{+}$obtained from $\Gamma$ via (15) and (16) are given by

$$
f_{-}(x)=f_{+}(x)= \begin{cases}x_{2} & \text { if } x_{2} \geq 0 \\ 0 & \text { if }-x_{1}^{2} \leq x_{2} \leq 0 \\ x_{2}+x_{1}^{2} & \text { if } x_{2}+x_{1}^{2} \leq 0 .\end{cases}
$$

they are quasiconvex, continuous but not g-pseudoconvex. Because the sandwich theorem there is no g-pseudoconvex function responding to the question of convex level sets integration of $\Gamma$.

The cyclic pseudomonotonicity and the maximal pseudomonotonicity of $\Gamma$ do not necessarily imply $f_{-}=f_{+}$or the uniqueness of a g-pseudoconvex function responding to the question as shown below

Example 6.2 Let $\widetilde{\Gamma}$ be defined by $\widetilde{\Gamma}\left(x_{1}, x_{2}\right):=\Gamma\left(x_{1}+1, x_{2}\right)$ where $\Gamma$ is as in the previous example. Let any nondecreasing function $k:[-1,0] \rightarrow[-1,0]$. Define $f$ on $\mathrm{IR}^{2}$ by

$$
f(x):= \begin{cases}x_{2}, & \text { if } x_{2} \geq 0, \\ x_{2}+\left(x_{1}+1\right)^{2}-1, & \text { if } x_{2}+\left(x_{1}+1\right)^{2} \leq 0, x_{2}<0, \\ x_{2}\left(x_{1}+1\right)^{-2}, & \text { if }-\left(x_{1}+1\right)^{2}<x_{2}<0, x_{1} \geq-1, \\ k\left(x_{2}\left(x_{1}+1\right)^{-2}\right), & \text { if }-\left(x_{1}+1\right)^{2}<x_{2}<0, x_{1}<-1 .\end{cases}
$$

Then, $f$ is solution of our problem. If $k(t)=-1$ for all $t \in[0,1]$ one obtains the lower bound $f_{-}$. If $k(t)=0$ for all $t \in[0,1]$ one obtains the upper bound $f_{+}$. If $k(t)=-(-t)^{\alpha}$ with $\alpha>0, f$ is g-pseudoconvex and $f\left(0, x_{2}\right)=x_{2}$ for all $x_{2}$. Different values of $\alpha$ lead to different g-pseudoconvex functions responding to the question of convex level sets integration of $\Gamma$.

\subsection{An Associated Implicit Differential Equation Problem}

Let $f$ be any quasiconvex function fulfilling both conditions (PM1) and (PM2). For $\lambda \in\left[\lambda_{-}, \lambda_{+}\right]$, let us define

$$
\theta_{\lambda}(y):=\sup [s: f(A y+s e)<\lambda], \quad A y+s e \in V .
$$

By construction,

$$
L_{\lambda}=\left\{x=A y+s e \in V: s \leq \theta_{\lambda}(y)\right\}=\operatorname{cl}(\{x \in V: f(x)<\lambda\}) .
$$


Therefore $\theta_{\lambda}$ is concave and continuous at any $y$ such that $A y+g_{\lambda}(y) e \in V$.

Let $x(y)=A y+\theta_{\lambda}(y) e$. If $f(x(y)) \geq \lambda$, then for any $x^{*}(y) \in \Gamma(x(y))$ one has

$$
\left\langle x^{*}(y), x^{\prime}-x(y)\right\rangle \leq 0 \quad \forall x^{\prime} \text { such that } f\left(x^{\prime}\right)<\lambda \leq f(x(y)) .
$$

Hence,

$$
\left\langle x^{*}(y), A y^{\prime}-A y\right\rangle+s^{\prime}-\theta_{\lambda}(y) \leq 0 \quad \forall s^{\prime} \leq \theta_{\lambda}\left(y^{\prime}\right),
$$

and therefore $-A^{\top} \Gamma(x(y)) \subset \partial\left(-\theta_{\lambda}\right)(y)$.

Assume now that $f(x(y))<\lambda$. Set $x_{k}=A y+\left(\theta_{\lambda}(y)+k^{-1}\right) e$ where $k$ is a positive integer. Then, $f\left(x_{k}\right) \geq \lambda$. Therefore for any $x_{k}^{*} \in \Gamma\left(x_{k}\right)$ one has

$$
\left\langle x_{k}^{*}, x^{\prime}-x_{k}\right\rangle \leq 0 \quad \forall x^{\prime}=A y^{\prime}+s^{\prime} e \text { such that } f\left(x^{\prime}\right)<\lambda \leq f\left(x_{k}\right) .
$$

In view of the continuity properties induced by the maximal pseudomonotonicity of $\Gamma$ (see the end of Section 4), the sequence $\left\{x_{k}^{*}\right\}$ has cluster points and these cluster points belong to $\Gamma(x(y))$. Let $x^{*}(y)$ be such a cluster point. Then, by continuity, $-A^{\top} \Gamma(x(y)) \subset \partial\left(-\theta_{\lambda}\right)(y)$.

Set $g_{\lambda}=-\theta_{\lambda}$. Then $g_{\lambda}$ is convex and $A^{\top} \Gamma(x(y)) \subset \partial g_{\lambda}(y)$.

Recall that a convex function is uniquely defined up to an additive constant as soon as, at each point, one element of its subdifferential is known. Therefore $g_{\lambda}$ is solution of the partial differential equation problem: Find $g_{\lambda}$ convex such that

$$
g_{\lambda}(0)=-\lambda, \quad \partial g_{\lambda}(y) \supset A^{\top} \Gamma\left(A y-g_{\lambda}(y) e\right) .
$$

Compare with (7). Here, cyclic and maximal cyclic monotonicity imply the existence of convex functions $g_{\lambda}$ that are solutions of the problem but, in contrast to the differentiable case discussed in section 4, without additional assumptions there is no uniqueness. See examples (6.1) and (6.2). Indeed $f_{-}$ and $f_{+}$generate different $\theta_{\lambda}$ and $g_{\lambda}$ when they differ.

\subsection{The Two Dimensional Case}

Let us seek conditions ensuring $f_{-}=f_{+}$. Let $d=A y \in \mathbb{R}^{n}$ such that $\|d\|=1$. Define

$$
E^{d}=\{x=t d+\lambda e \in V: \lambda, t \in \mathbb{R}\} .
$$

Next, let us introduce for all $x \in V \cap E^{d}$ the set

$$
J^{d}(x):=\left\{\begin{array}{c}
\exists\left(x_{0}, x_{0}^{*}\right),\left(x_{1}, x_{1}^{*}\right), \cdots,\left(x_{p}, x_{p}^{*}\right) \in \operatorname{graph}(\Gamma), \\
\left.y \in V \cap E^{d}: x_{i+1}-x_{i}\right\rangle \geq 0, i=0,1, \cdots, p-1, \\
x_{0}=x, x_{p}=y, x_{i} \in V \cap E^{d}, i=0,1, \cdots, p .
\end{array}\right\},
$$


and the functions

$$
\begin{aligned}
& f_{-}^{d}(x):=\sup \left[t \in\left[\lambda_{-}, \lambda_{+}\right]: x \in J^{d}(t e)\right], \\
& f_{+}^{d}(x):=\inf \left[t \in\left[\lambda_{-}, \lambda_{+}\right]: t e \in J^{d}(x)\right] .
\end{aligned}
$$

Clearly, $J^{d}(x) \subset J(x)$. Hence,

$$
f_{-}^{d}(x) \leq f_{-}(x) \leq f_{+}(x) \leq f_{+}^{d}(x) \quad \forall x \in V \cap E^{d}
$$

Proposition 6.1 Assume that $\Gamma$ is cyclically pseudomonotone and for all $d \in \mathbb{R}^{n}$ with $\langle e, d\rangle=0$ and $\|d\|=1$ it holds $f_{-}^{d}(x)=f_{+}^{d}(x) \quad \forall x \in V \cap E^{d}$. Then, there exists one and only one quasiconvex function on $V$ such that (PM1) and (PM2) hold.

Proof: Let any $x \in V$. There exist $d$ such that $\|d\|=1,\langle e, d\rangle=0$ and $t, \lambda \in \mathbb{I R}$ such that $x=t d+\lambda e$. Because $(22)$ one has $f_{-}(x)=f_{+}(x)$. Set $f(x)=f_{-}$. Then $f$ is quasiconvex, (PM1) and (PM2) hold. The uniqueness of such a function follows from the sandwich theorem.

Let us introduce the multivalued map $\Gamma^{d}: \mathrm{IR}^{2} \rightrightarrows \mathrm{IR}^{2}$ defined by

$$
\Gamma^{d}(t, \lambda)=\left(d^{\top} \Gamma(t d+\lambda e), 1\right)
$$

Then $\Gamma^{d}$ is cyclically pseudomonotone and maximal pseudomonotone if $\Gamma$ is so. Theorem 6.1 invites to study the two-dimensional case.

From (19), there exists a convex function $g_{\lambda}^{d}$ solution of the implicit equation

$$
g_{\lambda}^{d}(t)=-\lambda+\int_{0}^{t} h^{d}\left(r, g_{\lambda}^{d}(r)\right) d r,
$$

where $h^{d}(r, s) \in d^{\top} \Gamma(r d-s e)$. Is $g_{\lambda}^{d}$ uniquely defined? Classical criteria for uniqueness of solutions of differential equations (for the case $t>0$ ) are:

Lipschitz criterion: For any $\bar{r} \in[0, t]$ there is a neighborhood $W$ of $\left(\bar{r}, g_{\lambda}^{d}(\bar{r})\right)$ and $K>0$ such that

$$
\left|h^{d}\left(r, g_{\lambda}(r)+\rho\right)-h^{d}\left(r, g_{\lambda}(r)\right)\right| \leq K|\rho| \quad \forall\left(r, g_{\lambda}^{d}(r)+\rho\right) \in W .
$$

Nagumo criterion: For any $\bar{r} \in[0, t]$ there is a neighborhood $W$ of $\left(\bar{r}, g_{\lambda}^{d}(\bar{r})\right)$ and $K>0$ such that

$$
\left|h^{d}\left(r+\delta, g_{\lambda}(r)+\rho\right)-h^{d}\left(r, g_{\lambda}(r)\right)\right| \leq \frac{|K \rho|}{\delta} \forall\left(r+\delta, g_{\lambda}^{d}(r)+\rho\right) \in W, \delta>0 .
$$


The Nagumo criterion, if less known, is significantly stronger than the Lipschitz criterion. For a recent reference see [8]. Let us show that it implies uniqueness. Assume that $g_{\lambda}^{d}$ and $g_{\lambda}^{d}+\varepsilon$ are two solutions of (23). Let

$$
r=\sup \left[t: \varepsilon\left(t^{\prime}\right)=0 \forall t^{\prime} \in[0, t]\right] .
$$

Then, $\varepsilon(r)=0$ and for $t>r$,

$$
|\varepsilon(t)| \leq K \int_{0}^{t-r} \frac{|\varepsilon(s)|}{s-r} d s \leq \mu(t),
$$

where $\mu$ is the solution of the differential equation $(t-r) \mu^{\prime}(t)=K \mu(t)$ with the initial condition $\mu(r)=\varepsilon(r)=0$. Then $\mu(t)=\varepsilon(t)=0$ for $t>r$. The definitions of $r$ and $W$ imply $\varepsilon\left(t^{\prime}\right)=0$ for all $t^{\prime} \in[0, t]$ and therefore $g_{\lambda}^{d}$ and $g_{\lambda}^{d}+\varepsilon$ coincide on $[0, t]$.

Although these criteria are usually formulated for single-valued functions $h^{d}$, they are adapted to our case in taking into account the convexity of the function $g_{\lambda}^{d}$ and the continuity properties of the map $\Gamma$ induced by the maximal cyclic pseudomonotonicity. Take $h(r, s)=\max \left[\langle\gamma, d\rangle: \gamma \in d^{\top} \Gamma(r d-s e)\right]$. The two criteria are easily transposed to the $t<0$ case: take $d=-d$.

If the Nagumo criterion holds, then the function $g_{\lambda}^{d}$ in (23) is uniquely defined in the sense that it does not depend on the function $f$ fulfilling (PM1) and (PM2).

Clearly, both Lipschitz and Nagumo criteria are not fulfilled in examples (6.1) and (6.2).

\subsection{Existence and uniqueness}

Based on the Nagumo criterion, we consider the following condition:

Nagumo type condition: For all $\bar{x} \in V$, there exist $K(\bar{x})>0$ and $W(\bar{x})$ neighborhood of $\bar{x}$ such that for all $x_{1}, x_{2} \in W(x)$ with $e^{\top}\left(x_{1}-x_{2}\right) \neq 0$ it holds

$$
\min _{x_{i}^{*} \in \Gamma\left(x_{i}\right), i=1,2}\left|\left(x_{1}^{*}-x_{2}^{*}\right)^{\top} A A^{\top}\left(x_{1}-x_{2}\right)\right| \leq K(\bar{x}) \frac{\left\|A^{\top}\left(x_{1}-x_{2}\right)\right\|}{\left|e^{\top}\left(x_{1}-x_{2}\right)\right|} .
$$

The following proposition provides a weaker but more practicable condition

Proposition 6.2 Assume that for all $\bar{x} \in V$, there exist $K(\bar{x})>0$ and $W(\bar{x})$ neighborhood of $\bar{x}$ such that

$$
\left|\left\langle e, x_{1}-x_{2}\right\rangle\right|\left\|x_{1}^{*}-x_{2}^{*}\right\| \leq K(\bar{x}) \quad \forall x_{1}, x_{2} \in W(\bar{x}), \forall x_{i}^{*} \in \Gamma\left(x_{i}\right), i=1,2,(\widetilde{N})
$$

then the Nagumo type condition holds. 
Proof: On one hand, $\left|\left(x_{1}^{*}-x_{2}^{*}\right)^{\top} A A^{\top}\left(x_{1}-x_{2}\right)\right| \leq\left\|A^{\top}\left(x_{1}^{*}-x_{2}^{*}\right)\right\|\left\|A^{\top}\left(x_{1}-x_{2}\right)\right\|$. On the other hand, since $\left\langle e, x_{1}^{*}-x_{2}^{*}\right\rangle=0$, one has $\left\|A^{\top}\left(x_{1}^{*}-x_{2}^{*}\right)\right\|=\left\|x_{1}^{*}-x_{2}^{*}\right\|$. The result follows.

Assume that $(\mathrm{N})$ holds. Then, given $\lambda$, the two functions $\theta_{\lambda}, g_{\lambda}$ and the set $L_{\lambda}$ are uniquely defined. For all $y, y^{\prime} \in \operatorname{dom}\left(g_{\lambda}\right)$ it holds

$$
g_{\lambda}\left(y^{\prime}\right)-g_{\lambda}(y)=\int_{0}^{1}\left\langle A\left(y^{\prime}-y\right), \gamma(t)\right\rangle d t, \quad \gamma(t) \in \Gamma(x(t))
$$

where $x(t)=A\left(y+t\left(y^{\prime}-y\right)\right)-g_{\lambda}\left(y+t\left(y^{\prime}-y\right)\right) e$.

Next, given $x=A y-\mu e$, let us define a function $\xi$ as the solution of the implicit integration

$$
\xi(t)=\mu+\int_{0}^{t}\langle A(0-y), \gamma(t)\rangle d t, \quad \gamma(t) \in \Gamma(x(t)),
$$

where $x(t)=A(y-t y)-\xi(t) e$. This function is well defined. Take $\lambda=\xi(1)$. Then, in view of $(24), g_{\lambda}(y)=\mu$, and $x=A y-g_{\lambda}(y) e$.

Set $\bar{f}(x)=-\xi(1)$.

Theorem 6.4 If $\Gamma$ is maximal cyclically pseudomonotone and the Nagumotype condition holds, then $\bar{f}$ is the unique g-pseudoconvex, lower semicontinuous function such that $\bar{f}(\lambda e)=\lambda$ for all $\lambda \in[-\bar{\lambda}, \bar{\lambda}]$ and, for all $x \in V, \Gamma(x)$ generates $N(x, \bar{f})$ and $\widetilde{N}(x, \bar{f})$.

Proof: By construction $\bar{f}(\lambda e)=\lambda$. Let $f$ be any quasiconvex function which fulfills (PM1) and (PM2) (for instance $f_{-}$or $f_{+}$) and let any $\lambda$. Then,

$$
\{x \in V: \bar{f}(x) \leq \lambda\}=\operatorname{cl}(\{x \in V: f(x)<\lambda\}) .
$$

Hence, $\bar{f}$ is quasiconvex and lower semicontinuous. Because the functions $g_{\lambda}$ are uniquely defined, $\bar{f}\left(x^{\prime}\right)>\bar{f}(x)$ if $x^{\prime}=x+K$ and $\bar{f}\left(x^{\prime \prime}\right)<\bar{f}(x)$ if $x^{\prime \prime}=$ $x-K$. It follows that $\bar{f}$ is g-pseudoconvex. If $x^{*} \in \Gamma(x)$, then (18) implies that $\left\langle x^{*}, x^{\prime}-x\right\rangle \leq 0$ whenever $\bar{f}\left(x^{\prime}\right) \leq \bar{f}(x)$ and therefore $x^{*} \in \bar{\Gamma}(x) . \Gamma$ and $\bar{\Gamma}$ coincide because both are maximal.

\section{The Revealed Preference Problem}

Let us consider the case where a consumer determines his choice among a closed convex set of available goods $C \subset \mathrm{IR}_{+}^{n}$ via a preorder of preferences in taking into account his budget $b>0$ and the vector of unit prices of goods $p \in \mathrm{IR}_{+}^{n}$. The preorder enjoys the following properties: 
The preorder is total: Given $x, y \in C$, one and only one of the three following relations holds: $x \succ y$ ( $x$ strictly preferred to $y), y \succ x$ ( $y$ strictly preferred to $x$ ) and $x \sim y$ ( $x$ and $y$ are equivalent).

The preorder is transitive: If $x$ is preferred to $y$ and $y$ is preferred to $z$ then $x$ is preferred to $z$. If in addition $x \succ y$ or $y \succ z$, then $x \succ z$.

Non satiety property: $x \succ y$ whenever $x \neq y$ with $x_{i} \geq y_{i}$ for $i=1, \cdots n$.

The preorder is continuous: For all $x \in C$, the sets $S(x)=\{y \in C: y \succeq x\}$ and $C(x)=\{y \in C: y \sim x\}$ are closed.

The consumer problem consists in finding $x \in C$ fulfilling the constraint budget $\langle p, x\rangle \leq b$ such that there is no $y$ such that $\langle p, y\rangle \leq b$ with $y \succ x$. Denote by $D(p, b)$ the set of such $x$. If $x \in D(p, b)$, then $\langle p, x\rangle=b$ because of the non satiety property. The map $D$ is called the demand associated to the preorder. The revealed preference problem consists of recovering the preorder from the demand $D$. It is clear that

$$
\begin{aligned}
& \widetilde{S}(x)=\{y \in C: y \succ x\} \subset S(x)=\{y \in C:\langle p, x\rangle>b\}, \\
& S(x)=\{y \in C: y \succeq x\} \subset S(x)=\{y \in C:\langle p, x\rangle \geq b\} .
\end{aligned}
$$

Therefore $p$ belongs to the normal cones at $x$ to the level sets $S(x)$ and $\widetilde{S}(x)$. The revealed preference problem is a level sets integration problem. It consists of finding a utility function $u$ associated to the preorder: $x \succeq y$ if and only if $u(x) \geq u(y)$.

The revealed preference problem has been initiated by Samuelson [9] and Houthakker [10], see also Afriat [11], Hurwicz-Uzawa [12], Varian [13]. A recent reference is Crouzeix-Eberhard [14]. The strong axiom of revealed preferences traduces the transitivity of the preorder. It corresponds exactly to the cyclic pseudomonotonicity of the map $-P$ where $P(x)=\{p: x \in D(p, 1)\}$. Our paper responds to the question: the axioms of the preorder (including the strong axiom of revealed preferences) together with the maximal cyclic pseudoconvexity of the map $-P$ are not sufficient to ensure the existence of a corresponding utility. An additional Nagumo-type condition is required.

\section{Conclusion}

The maximal cyclic monotonicity of a multivalued map is sufficient to ensure the existence and uniqueness up to a constant of a convex function whose subdifferential coincides with the map. In this paper we have shown that maximal cyclic pseudo monotonicity is not sufficient to ensure both existence and uniqueness up to a scalarization of a pseudoconvex function whose the normal cones are generated by the map. An additional condition is needed. Also, we 
have responded to the unsolved question: different formulations of the strong revealed preferences axiom are not sufficient to ensure the uniqueness of a preference preorder associated with a demand relation.

\section{References}

1. Crouzeix J.-P., Rapcsák T.: Integrability of pseudomonotone differentiable maps and the revealed preference problem. J. Conv. Anal. 12, 431-446 (2005)

2. Rockafellar R. T. : Convex Analysis. Princeton University Press, Princeton, New Jersey (1970)

3. Crouzeix J.-P., Ocaña E.: Maximality is nothing but continuity. J. Conv. Anal. 17, 521-534 (2010)

4. Crouzeix J.-P., Ferland J.A.: Criteria for differentiable generalized monotone maps. Math. Program. 75, 399-406 (1996)

5. Crouzeix J.-P., Ferland J.A.: Criteria for quasiconvexity and pseudoconvexity : Relationships and Comparisons. Math. Program. 23, 193-205 (1982)

6. Crouzeix J.-P., Eberhard A., Ralph D.: A geometrical insight on pseudoconvexity and pseudomonotonicity. Math. Program. Ser. B 123, 61-83 (2010)

7. Crouzeix J.-P., Keraghel A., Rahmani N.: Integration of pseudomonotone maps and the revealed preference problem. Optimization 60, 783-800 (2011)

8. Constantin A.: On Nagumo's theorem. Proc. Japan Acad. Math. Sci. Ser. A 86, 41-44 (2010)

9. Samuelson P.A.: Consumption theory in terms of revealed preference. Economica, 243-253 (1948)

10. Houthakker H.S.: Revealed Preferences and the Utility Function. Economica, 159-174 (1950)

11. Afriat S.N.: The construction of a utility function from expenditure data. Int. Econ. Rev. 8, 67-77 (1967)

12. Hurwicz L., Uzawa H.: On the Integrability of Demand Functions. Preference Utility and Demand, Harcourt Brace Jovanovich, New York, 114-148 (1971)

13. Varian H.R.: The non-parametric approach to demand analysis. Econometrica 50, 945-974 (1982)

14. Crouzeix J.-P., Eberhard A.: Existence of Closed Graph, Maximal, Cyclic PseudoMonotone Relations and Revealed Preference Theory. J. Ind. Man. Optimization 3, 233-255 (2007) 